\title{
Estudo de tecnologias de investigação ambiental em alta resolução para refinamento do modelo conceitual - estudo de caso: Duque de Caxias (RJ), Brasil
}

\author{
Study of high-resolution site characterization technologies for the improvement \\ of the conceptual site model - case study: Duque de Caxias (RJ), Brazil
}

Mariana Gava Milani* $\odot$, Ana Cristina Malheiros Gonçalves Carvalho' ${ }^{\circledR}$

\begin{abstract}
RESUMO
Este trabalho apresenta os resultados da utilização de duas técnicas de investigação geoambiental em alta resolução: investigação passiva de vapores do solo e investigação com o uso de Membrane Interface Probe (MIP), em uma área contaminada por hidrocarbonetos de petróleo, localizada no município de Duque de Caxias, Rio de Janeiro. O processo de gerenciamento ambiental da área iniciou-se em 2012 e contemplou as etapas preconizadas nas legislações vigentes. Os resultados obtidos a partir das investigações tradicionais foram insuficientes para a compreensão das características físicas e geoquímicas necessária para o sucesso das fases de diagnóstico e, consequentemente, da remediação. Portanto, duas investigações em alta resolução foram conduzidas com o objetivo de refinar o modelo conceitual de forma a atender adequadamente à Resolução CONAMA no 420/09 e à Resolução CONEMA no 44/12, permitindo ações futuras mais eficientes. A investigação passiva de vapores do solo utilizou amostradores compostos por materiais adsorventes granulares, encapsulados em uma membrana microporosa hidrofóbica e quimicamente inerte que permite a difusão dos vapores presentes no meio. Os resultados representam qualitativamente a presença de contaminação no subsolo. O MIP é uma ferramenta de direct push com medição em tempo real, que detecta a presença da contaminação tanto em meios insaturados quanto saturados inconsolidados. A partir dos resultados integrados, foi possível constatar que a distribuição de compostos orgânicos voláteis (volatile organic compounds (VOCs)) em água subterrânea ocorre de forma descontínua ao longo da área, sendo possível identificar cinco hotspots distintos e suas diferentes áreas fonte, incluindo uma região com presença de fase livre.
\end{abstract}

Palavras-chave: investigação em alta resolução; áreas contaminadas; hidrocarbonetos derivados de petróleo; modelo conceitual.

\begin{abstract}
This paper presents the results of two high-resolution site characterization (HRSC) techniques: passive investigation of soil vapors and investigation using Membrane Interface Probe (MIP), in an area contaminated by petroleum hydrocarbon, located in the municipality of Duque de Caxias, Rio de Janeiro. The environmental management process in the area began in 2012 and contemplated the stages recommended by the current legislation. The results obtained from the traditional investigations were insufficient for the understanding of the physical and geochemical aspects for the success of the diagnostic phases and, consequently, the remediation phase. Therefore, it was conducted two high-resolution investigations with the objective of improving the conceptual model in order to comply adequately with CONAMA Resolution 420/09 and CONEMA Resolution 44/12, allowing future actions more efficient. The soil gas passive investigation used samplers composed of granular adsorbent materials, encapsulated in a hydrophobic and chemically inert microporous membrane that allows the diffusion of the vapors present in the media. The results qualitatively represent the presence of contamination in the subsoil. MIP is a direct push tool with real-time measurement, which detect the presence of contamination in both unsaturated and saturated media. From the integrated results, it was possible to verify that the distribution of volatile organic compounds (VOCs) in groundwater occurs in a discontinuous way throughout the area. It was possible to identify five distinct hotspots and their different source areas, including a region with the presence of free-phase.
\end{abstract} Keywords: high-resolution site characterization; contaminated areas; petroleum hydrocarbons; conceptual site model.

'Pontifícia Universidade Católica - Rio de Janeiro (RJ), Brasil.

*Autora correspondente: marianagmilani@gmail.com

Conflitos de interesse: os autores declaram não haver conflito de interesses.

Financiamento: nenhum.

Recebido: 12/O2/2019 - Aceito: 28/02/2O2O - Reg. ABES: 20190043 


\section{INTRODUÇÃO}

Uma área contaminada (AC) é considerada uma situação de risco pois sua existência pode gerar diversos problemas, como danos à saúde, comprometimento da qualidade dos recursos hídricos, restrições ao uso do solo e danos ao patrimônio público e privado, desvalorização de propriedades, além de danos ao meio ambiente (CETESB, 2013).

A problemática das ACs começou a ser estudada há aproximadamente 30 anos, com o surgimento de ocorrências como o caso do Love Canal, localizado em Niagara Falls, Nova York, Estados Unidos, e o caso de Lekkerkerk, localizado em Roterdã, Holanda (RODRIGUES JR., 2003). O caso do Love Canal se tornou conhecido mundialmente após ser revelado que o local foi utilizado para enterrar 21 mil tonéis contendo lixo tóxico pela Hooker Chemical Company (hoje Occidental Petroleum Corporation), e foi uma das principais razões que levaram o Congresso Americano a aprovar a Lei do Superfund (RODRIGUES JR., 2003). Este é um fundo que permite à Environmental Protection Agency (EPA) remediar sites contaminados quando um responsável legal viável não é existente, além de obrigar as partes responsáveis pela contaminação a realizar ações de remediação ou reembolsar o governo pelo trabalho de remediação liderado pela EPA (USEPA, 2019). No caso de Lekkerkerk, foram encontrados resíduos de diferentes atividades industriais enterrados em área residencial, acarretando contaminação do subsolo, o que levou a Holanda a ser um dos primeiros países europeus a adotar uma legislação específica sobre proteção e contaminação do solo (RODRIGUES JR., 2003). De acordo com Sánchez (1998), em ambos os casos houve um gasto enorme para a recuperação das áreas: cerca de 250 milhões de dólares no caso do Love Canal e de 65 milhões de dólares no caso de Lekkerkerk.

No Brasil, podem ser citados como exemplos de ocorrências de ACs de grande repercussão e danos ambientais o caso da fábrica de pesticidas do antigo Ministério da Educação e Saúde que operou entre os anos de 1950 e 1956 no município de Duque de Caxias, Rio de Janeiro, no qual foram identificadas 40 toneladas de pesticida (i.e., hexaclorociclohexano) abandonadas na área da Cidade dos Meninos; o caso do antigo lixão da Companhia Siderúrgica Nacional (CSN) localizado em Volta Redonda, Rio de Janeiro, no qual foram identificados resíduos industriais dispostos de forma irregular, afetando áreas de uso residencial; entre outros.

O gerenciamento de ACs no Brasil deve seguir as etapas preconizadas na Resolução do Conselho Nacional do Meio Ambiente (CONAMA) no 420 de 28 de dezembro de 2009 em nível federal (BRASIL, 2009), e na Resolução do Conselho Estadual do Meio Ambiente (CONEMA) n ${ }^{\circ} 44$ de 14 de dezembro de 2012 em nível estadual (estado do Rio de Janeiro) (CONEMA, 2012), e inclui as etapas de identificação, diagnóstico e intervenção.

As atividades desenvolvidas nas etapas de diagnóstico dão origem ao modelo conceitual do site (Conceptual Site Model (CSM)), que é uma ferramenta de planejamento que organiza as informações que já foram obtidas sobre a área de interesse e que identifica as informações adicionais necessárias para apoiar as decisões de forma a se atingir os objetivos do projeto (USEPA, 2010).

As etapas de identificação e diagnóstico são usualmente conduzidas no Brasil por métodos de investigação consagrados e obrigatórios (investigação tradicional), que nem sempre conseguem proporcionar a compreensão dos aspectos físicos e geoquímicos da área de interesse (o site) necessária para se obter sucesso nas fases de identificação e diagnóstico e, consequentemente, de remediação. Isso ocorre principalmente quando há presença de fase livre (i.e., fase líquida não aquosa, cujas diferenças nas propriedades físicas e químicas dos contaminantes com a água resultam na imiscibilidade em água - NEWELL et al., 1995 apud MOREIRA; DOURADO, 2005), devido a sua forma característica de movimentação, acumulação e adsorção ao solo.

Além disso, em função principalmente de fatores financeiros e de planejamento, muitas vezes essas etapas são negligenciadas, ocasionando uma má caracterização da área. Investigações inadequadas acarretam um modelo conceitual muito simplificado (SANTOS et al., 2015), ocasionando um mau gerenciamento ambiental da área e o não atendimento das legislações vigentes. Dessa forma, é importante recorrer às abordagens e técnicas de investigação mais eficientes, a exemplo das técnicas de investigação em alta resolução (High Resolution Site Characterization (HRSC)).

As técnicas de HRSC ainda são pouco aplicadas no Brasil devido à falta de conhecimento sobre as tecnologias disponíveis e ao baixo nível de exigência dos órgãos ambientais. A Companhia Ambiental do Estado de São Paulo (CETESB) foi a primeira agência ambiental a recomendar a utilização de métodos de investigação em alta resolução em sua Decisão de Diretoria (DD) no 38 de 7 de fevereiro de 2017 (CETESB, 2017), porém não existem no Brasil diretrizes relacionadas a esses métodos, ou técnicas, de investigação.

Segundo Riyis et al. (2013), ferramentas de alta resolução associadas a ferramentas tradicionais proporcionam uma economia significativa de tempo, permitem um entendimento adequado do meio físico e a elaboração de um modelo conceitual do site sólido e em tempo real, que pode subsidiar projetos de remediação baseados em dados mais confiáveis que aqueles que seriam obtidos em investigações tradicionais, preocupadas somente em seguir as regras estabelecidas pelos órgãos ambientais. Adicionalmente, segundo estudo desenvolvido por Santos et al. (2015), verificou-se que o uso de uma ferramenta de alta resolução permitiu um avanço no modelo conceitual da área estudada, o que reduziu a necessidade de eventuais investigações adicionais, possibilitando maior eficiência e eficácia nas etapas de remediação.

Porém, existem diversas técnicas de HRSC (por exemplo: AGI EarthImager 3D; passive soil gas (PSG) Samplers; Membrane Interface Probe (MIP); Hydraulic Profiling Tool (HPT); poços de monitoramento (CMT)), algumas ainda relativamente caras no Brasil. Dessa forma, é importante entendê-las e utilizá-las em conjunto, principalmente quando uma ferramenta pode otimizar o uso de outra com custo mais alto.

Nesse contexto, este estudo tem como objetivo principal obter um modelo conceitual mais representativo de uma área de interesse. Para atingir esse objetivo, foram utilizadas duas técnicas de investigação em alta resolução, de forma complementar: investigação passiva de vapores do solo e investigação com uso de MIP.

\section{Gerenciamento de áreas contaminadas}

Uma contaminação pode estar relacionada a atitudes negligentes e/ou liberações involuntárias de substâncias químicas perigosas ou de subprodutos gerados por estas (RODRIGUES JR., 2003). Existem inúmeros contaminantes passíveis de serem encontrados em solo e água subterrânea, como os hidrocarbonetos derivados de petróleo, e o nível de complexidade de uma AC pode variar de diversas formas. Os contaminantes podem, por exemplo, migrar do solo para a água subterrânea, ou serem carreados para áreas no entorno e/ou cursos d'água por meio da lixiviação dos contaminantes ou pela poeira. 
O gerenciamento de ACs no estado do Rio de Janeiro encontra-se inserido no âmbito do licenciamento ambiental, sob a diretoria de Licenciamento Ambiental, atualmente por meio do Decreto Estadual no 44.820 de 2014. As Licença de Operação e Recuperação (LOR) e Licença Ambiental de Recuperação (LAR), além do Termo de Encerramento, instituídas pelo Decreto no 42.159 de 2009 (que também instituiu o Sistema de Licenciamento Ambiental (SLAM)), inserem a investigação da qualidade do solo e água subterrânea no processo de licenciamento. Em 2014, os conceitos e procedimentos do SLAM foram revisados por meio do Decreto Estadual no 44.820 .

A legislação específica para ACs vigente no estado do Rio de Janeiro (exceto para posto de serviço, que conta com legislação própria) é a Resolução CONEMA no 44 de 14 de dezembro de 2012. A Resolução CONEMA nº 44/12 dispõe sobre a obrigatoriedade da identificação de eventual contaminação ambiental do solo e das águas subterrâneas por agentes químicos, no processo de licenciamento ambiental estadual.

A maior parte dos conceitos da Resolução CONEMA nº 44/12 é baseada na Resolução CONAMA no 420/09. Além disso, os valores orientadores estabelecidos pela Resolução CONEMA n ${ }^{\circ}$ 44/12 para o estado do Rio de Janeiro são os valores orientadores listados no Anexo II da Resolução CONAMA nº 420/09. A Resolução CONEMA no 44/12 estabelece um fluxograma de gerenciamento de Acs. Um fluxograma simplificado é apresentado na Figura 1.

De acordo com a Resolução CONEMA n 44/12, devem ser levadas em consideração a Resolução CONAMA n $420 / 09$ e as normas técnicas vigentes da Associação Brasileira de Normas Técnicas (ABNT) referentes ao gerenciamento de ACs. Observa-se que as principais normas técnicas vigentes atualmente no Brasil e específicas de ACs dão diretrizes principalmente para métodos de investigação tradicionais, que são as normas usualmente exigidas pelo Instituto Estadual do Ambiente (INEA) no processo de gerenciamento de ACs.

O gerenciamento de ACs inicia-se com a etapa de identificação das áreas suspeitas de contaminação (AS) e áreas com potencial de contaminação (AP); nas etapas seguintes, busca-se obter a delimitação horizontal e vertical das plumas de contaminação, quando necessário, assim como um maior entendimento do comportamento do fluxo de água subterrânea e dos contaminantes na área de interesse. Porém, isso nem sempre é alcançado nessa etapa, dependendo

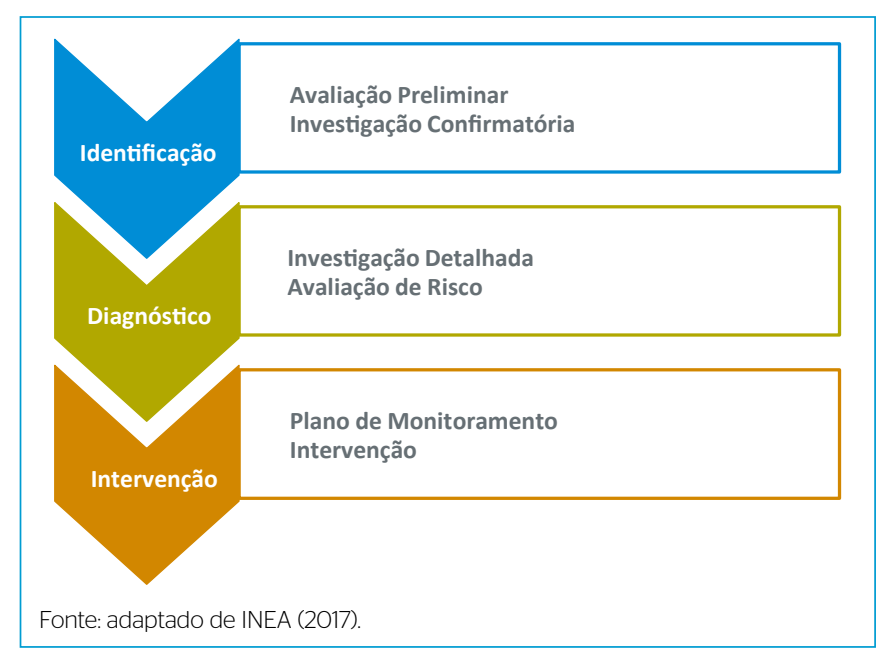

Figura 1 - Gerenciamento de áreas contaminadas no estado do Rio de Janeiro: fluxograma simplificado. das características da área sob investigação, dos contaminantes presentes e dos métodos de investigação utilizados.

ACs com presença de fase líquida leve não aquosa (Light Non-Aqueous Phase Liquid (LNAPL)) podem permanecer sob investigação e/ou em fase de remediação por anos sem obter um resultado de risco aceitável, pois as investigações por meio de métodos tradicionais em muitos casos não são suficientes para a detecção de toda a extensão do LNAPL, como foi mencionado anteriormente. Em alguns casos, a presença de LNAPL pode não ser identificada a partir de métodos tradicionais, principalmente em casos nos quais as fontes de contaminação são desconhecidas. Nesses casos, o LNAPL pode permanecer atuando como fonte de contaminação secundária por um longo período de tempo, contaminando grandes volumes de solo e água subterrânea. Portanto, ressalta-se a importância da aplicação de métodos alternativos de investigação, para que ações de contenção e remediação possam ser aplicadas em curto prazo, evitando assim o espalhamento da contaminação e a exposição de mais receptores humanos e/ou ecológicos à essa contaminação.

\section{Contaminantes orgânicos derivados do petróleo}

O uso crescente de derivados de petróleo em processos industriais proporciona a geração de uma diversidade de produtos cada vez mais utilizados pela sociedade atual. Uma das consequências negativas do uso e da manipulação de hidrocarbonetos é a geração de resíduos e efluentes altamente poluidores (MOREIRA; DOURADO, 2005). A destinação desses resíduos é um dos principais problemas ambientais da atualidade, pois muitas vezes não existem opções de reutilização, e o transporte e a disposição em locais adequados demandam elevados investimentos. Procedimentos inadequados, como o descarte indevido, resultam em ações danosas ao meio ambiente, contaminando tanto o solo quanto os recursos hídricos, com graves consequências, destacando-se o risco de exposição a esses resíduos tanto por receptores humanos como ecológicos (MOREIRA; DOURADO, 2005).

Os contaminantes podem migrar do solo para a água subterrânea, ou podem ser carreados para áreas no entorno e/ou cursos d'água por meio da lixiviação dos contaminantes ou pela poeira. O nível de complexidade de uma AC pode variar de diversas formas, por exemplo, uma área pode apresentar mais de um tipo de contaminante, pode apresentar mais de um meio físico contaminado, e a contaminação pode afetar diversas comunidades incluindo receptores mais sensíveis como crianças e idosos (DIAZ, 1999 apud RODRIGUES JR., 2003).

Um dos problemas de contaminação de águas subterrâneas mais comum está relacionado à presença de LNAPL, resultantes da liberação de produtos derivados de petróleo no meio ambiente, que são caracterizados por serem menos densos do que a água.

Os LNAPLs estão presentes no subsolo em inúmeras áreas sob investigação em todo o mundo e são frequentemente o foco de esforços de investigação e remediação. Em geral, LNAPLs em fase livre e fase residual são fontes secundárias de contaminação da água subterrânea, do solo e do ar dos poros, a médio e longo prazo, causando uma grande preocupação devido à sua persistência no subsolo e habilidade de contaminar grandes volumes de solo e água subterrânea (KIM; CORAPCIOGLU, 2003).

Entre os compostos químicos tipicamente encontrados em LNAPL, benzeno, tolueno, etilbenzeno e xilenos (identificados como BTEX) têm grande importância ambiental, destacando-se o benzeno, por ser considerado o mais tóxico. O benzeno é classificado como um composto carcinogênico confirmado, 
com evidências de carcinogenicidade a partir de todas as rotas de exposição em animais e humanos (IARC, 2017).

As investigações por meio de métodos tradicionais em muitos casos não são suficientes para a detecção da presença de LNAPL e/ou de toda a extensão da contaminação, mantendo ACs com presença de LNAPL sob investigação e/ou em fase de remediação por anos sem obter um resultado de risco aceitável.

Após décadas de experiência em remediação de áreas com presença de LNAPL, a percepção de como gerenciar esse problema mudou (SUTHERSAN; KOONS; SCHNOBRICH, 2015) e novos métodos de investigação foram surgindo, como as técnicas de investigação em alta resolução, que detectam contaminação de forma mais rápida e com maior precisão, para que ações de contenção e remediação possam ser aplicadas em curto prazo, evitando assim o espalhamento da contaminação e a exposição de mais receptores humanos e/ou ecológicos a essa contaminação.

\section{METODOLOGIA}

As técnicas de HRSC incluem o uso de ferramentas de coleta de dados com menor tempo de resposta (muitas delas sendo tecnologias de medição em tempo real) e mais precisas, quando comparadas com a investigação tradicional, e possibilitam a utilização de medições e densidades de amostragem mais apropriadas à complexidade do site e da contaminação, diminuindo a incerteza de amostragem, no que diz respeito à definição das distribuições dos contaminantes e do contexto do meio físico no qual estão presentes. Com um grau de incerteza menor, consegue-se subsidiar uma remediação mais rápida e eficaz.

HRSC é uma prática que faz parte da abordagem de gerenciamento de ACs desenvolvida pela Agência de Proteção Ambiental Americana (United States Environmental Protection Agency (USEPA)) chamado de Triad Approach (Abordagem Tríade), cujo objetivo é gerenciar as incertezas, aumentando a confiança de que as decisões do projeto, relacionadas à presença de contaminantes, localização, destino, exposição e opções para redução do risco, sejam feitas de forma correta, economicamente viável e efetiva (USEPA, 2004). A Abordagem Tríade é uma iniciativa tecnológica e científica, e não uma norma e/ou legislação.

O CSM é o produto primário da fase de diagnóstico da Abordagem Tríade e é a base fundamental para qualquer tomada de decisão realizada no gerenciamento de uma AC (USEPA, 2004).

\section{Os elementos da Abordagem Tríade são:}

- planejamento sistemático ou estratégico, para garantir a confiabilidade das tomadas de decisão;

- estratégias dinâmicas de trabalho, para permitir que os projetos sejam realizados da forma mais rápida e com menor custo, o que não seria alcançado com o uso de métodos tradicionais de gerenciamento de ACs;

- uso de tecnologias de medição em tempo real, o que torna as estratégias de trabalho mais dinâmicas, e dá suporte a tomadas de decisão em tempo real (USEPA, 2004)

As técnicas de HRSC fazem parte do terceiro vértice da abordagem tríade. O objetivo principal das técnicas de HRSC é identificar de forma rápida e eficiente as áreas impactadas, permitindo que as ações futuras e decisões sobre a remediação da área sejam feitas de forma mais efetiva (COUTINHO, 2002). As técnicas de HRSC não têm o intuito de substituir por completo as investigações tradicionais, mas sim de garantir uma maior exatidão na determinação da localização dos poços de monitoramento a serem instalados posteriormente para confirmação da contaminação, e possibilitar a instalação de um número reduzido de poços.

Para atingir o objetivo principal deste estudo foram aplicadas duas técnicas de investigação em alta resolução, de forma complementar: investigação passiva de vapores do solo e investigação com uso de MIP.

\section{Investigação passiva de vapores do solo}

A investigação passiva de vapores do solo é uma técnica de caracterização química, de varredura (ou screening) de compostos orgânicos voláteis (VOCs) e/ou compostos orgânicos semi-voláteis (SVOCs).

Nesse tipo de investigação, são instalados amostradores em sondagens com diâmetro variando de 1 a 1,5 polegada (pol), e com a profundidade variando de 0,10 a 1 metro $(\mathrm{m})$ de profundidade, devendo ser acima da zona saturada, em malhas variando de 10 até $40 \mathrm{~m}$ de espaçamento entre cada amostrador, dependendo do objetivo e do tamanho da área de interesse. $\mathrm{O}$ tempo de residência do amostrador no subsolo pode variar de 7 a 14 dias dependendo das características do site e objetivo do estudo.

Os tipos de contaminação detectados por essa tecnologia incluem derivados de petróleo, solventes, compostos organoclorados, nitroaromáticos, álcoois, explosivos, pesticidas, mercúrio, entre outros. Caso tais compostos estejam presentes nas proximidades do amostrador, eles difundem-se pela membrana e são adsorvidos ao material dos amostradores.

\section{Investigação com uso de membrane interface probe}

O MIP é uma ferramenta de direct push com medição direta em tempo real, que fornece dados contínuos sobre a distribuição de VOCs na subsuperfície, tanto em meios não saturados quanto saturados inconsolidados.

A sonda possui aproximadamente 60 centímetros $(\mathrm{cm})$ de comprimento e 57 milímetros ( $\mathrm{mm}$ ) de diâmetro e pode contemplar sistemas distintos: o MIP, utilizado para mapear a presença dos compostos de interesse; o Hydraulic Profiling Tool (HPT), que coleta informações de permeabilidade hidráulica subsuperficial do solo; e o dispositivo de Condutividade Elétrica (Electrical Conductivity (EC)), utilizado para medir a condutividade elétrica do solo.

A sonda do MIP é equipada com uma membrana porosa de politetrafluoretileno acoplada a uma chapa de aço inoxidável, a qual é aquecida eletricamente até a faixa ótima de temperatura (i.e., de 100 a 120 graus Celsius $\left({ }^{\circ} \mathrm{C}\right)$ ) para estimular o particionamento dos VOCs presentes no solo e/ou na água subterrânea próxima à ponta da sonda, fazendo com que esses compostos se volatilizem (Figura 2). Os VOCs, até um determinado tamanho molecular (i.e., moléculas do tamanho do naftaleno ou menores), difundem-se pela membrana, sob o efeito de um gradiente de concentração e são transportados por um gás de arraste (i.e., nitrogênio) por uma alça de tubulação (i.e., linha tronco). Os compostos orgânicos são carregados até a superfície, onde passam por três sensores químicos:

- Detector de fotoionização (PID), que consiste em uma lâmpada ultravioleta (UV) de 10,2 elétron volt (eV), que ioniza compostos com potencial de ionização menor do que 10,2 eV, incluindo compostos orgânicos aromáticos (por exemplo: benzeno, tolueno, etilbenzeno, xilenos), compostos clorados que possuem átomos de carbono em ligação dupla (Carbono $(\mathrm{C})=\mathrm{C}$ ), como tricloroeteno (TCE) e tetracloroeteno (PCE), entre outros, montada em uma célula eletrolítica termostaticamente controlada, de baixo volume e fluxo contínuo. 


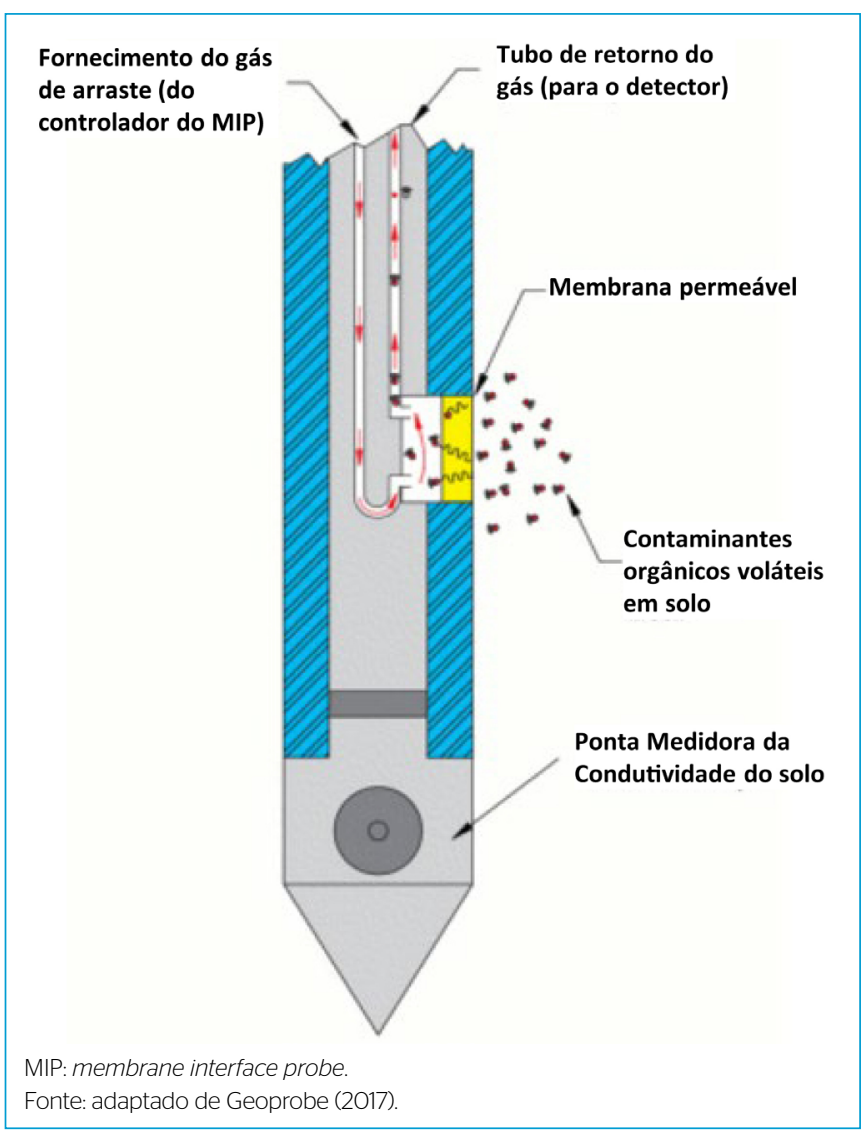

Figura 2 - Princípio da operação do membrane interface probe.

- Detector de compostos halogenados específicos (XSD), que consiste em uma sonda de cerâmica, um fio de platina (ânodo) e um grânulo de platina (cátodo) montado no interior de um reator de alta temperatura, sensível a átomos de halogêneo, incluindo bromo, cloro e flúor.

- Detector de ionização de chama (FID), que utiliza uma chama de hidrogênio para promover a combustão dos compostos dentro do gás de arraste para medição dos hidrocarbonetos de cadeia linear (por exemplo, metano) e concentrações elevadas de alguns compostos clorados.

As respostas dos detectores são reportadas em microvolts $(\mu \mathrm{V})$, representando as concentrações relativas totais de VOCs.

A sonda também conta com um conjunto de dipolos em sua ponta para medição de EC do solo e eventuais fluidos, na unidade de milisiemens por metro $\left(\mathrm{mS} . \mathrm{m}^{-1}\right)$, que pode ser usado como indicação de mudanças estratigráficas no ambiente em tempo real. A presença de ânions, como o cloreto, pode dificultar a interpretação das detecções de EC.

O sistema HPT cria perfis das propriedades hidráulicas do solo, que podem ser utilizadas para mensurar a permeabilidade e a condutividade hidráulica do local.

A mensuração da pressão estática (teste de dissipação) também pode ser realizada quando a sonda é paralisada em intervalos discretos, e proporciona uma estimativa do nível do lençol freático (nível d’água), com base na carga hidráulica imposta sobre a sonda em repouso comparada com a pressão medida na superfície antes de cada sondagem.
Em geral, a eficiência dessa técnica depende do tipo de solo, da concentração do contaminante, da saturação, da volatilidade do contaminante, entre outros.

Os dados de saída do MIP incluem até seis gráficos para cada ponto: três gráficos representam as respostas dos detectores químicos: PID, FID e XSD, medidos em $\mu \mathrm{V}$, e indicam as concentrações relativas da contaminação presente naquele ponto; um quarto gráfico apresenta a pressão do HPT em quilopascal $(\mathrm{kPa})$ e a vazão em mililitros por minuto $\left(\mathrm{mL} \cdot \mathrm{min}^{-1}\right)$; um quinto gráfico exibe as respostas do $\mathrm{EC}$ em $\mathrm{mS} . \mathrm{m}^{-1}$; um sexto gráfico é apresentado quando o teste de dissipação é realizado e mostra a estimativa do valor da condutividade (K) da formação, em metro por dia ( $\left.m \cdot d^{-1}{ }^{-1}\right)$, assim como o nível estático do lençol freático. Em todos os gráficos, o eixo Y é referente à profundidade.

\section{ESTUDO DE CASO}

A área de interesse é uma subárea de uma planta industrial localizada na borda noroeste da Baía de Guanabara, no município de Duque de Caxias, estado do Rio de Janeiro, Brasil.

A fim de se cumprir as etapas obrigatórias preconizadas na legislação vigente do estado do Rio de Janeiro do gerenciamento de ACs, uma investigação preliminar foi conduzida em agosto de 2013 na planta como um todo, uma investigação confirmatória foi realizada em fevereiro de 2014 e uma investigação detalhada em dezembro de 2014.

Duas áreas com potencial de contaminação (APs) foram identificadas na área de interesse (subárea da planta) durante a condução da investigação preliminar em 2013: a área de tancagem de produto derivado de petróleo e parque de bombas, e a área da torre de stripper que faz parte do sistema de tratamento de efluentes da planta.

Com base nas APs identificadas, 14 poços de monitoramento foram instalados na área de interesse, sendo 1 preexistente, instalado em 2012 como parte de uma investigação emergencial realizada na planta, 6 poços de monitoramento (PM-43 a 48) instalados na investigação confirmatória, e 7 poços de monitoramento (PM-74 a 80) instalados na investigação detalhada.

Todos os poços de monitoramento foram instalados para serem representativos do aquífero freático. Na Figura 3, é apresentada a pluma em fase dissolvida de benzeno do monitoramento realizado na área de interesse em março de 2016, prévio à investigação em alta resolução. As plumas de contaminação apresentadas neste estudo foram elaboradas no software AutoCAD ${ }^{\circledR}$ da Autodesk, a partir de faixas de concentração predeterminadas para representar as concentrações identificadas em água subterrânea nos poços de monitoramento existentes.

Observa-se que, após a condução das investigações tradicionais em um período de quatro anos, que incluíram a instalação de 14 poços de monitoramento e a condução de monitoramentos semestrais da água subterrânea, não foi possível delimitar as plumas de contaminação e/ou identificar todas as fontes da contaminação, incluindo possíveis fontes ativas.

Para alcançar a delimitação das plumas e a identificação de todos os hotspots continuando com a abordagem da investigação tradicional, considerando-se que a área de interesse possui aproximadamente $73.000 \mathrm{~m}^{2}$ de área, e a mobilidade do benzeno em água subterrânea que pode ser observada pela variação da pluma a cada campanha de monitoramento, o nível de incerteza quanto ao número de novos poços de monitoramento necessários e quanto às localizações dos mesmos pode ser considerado alto. 


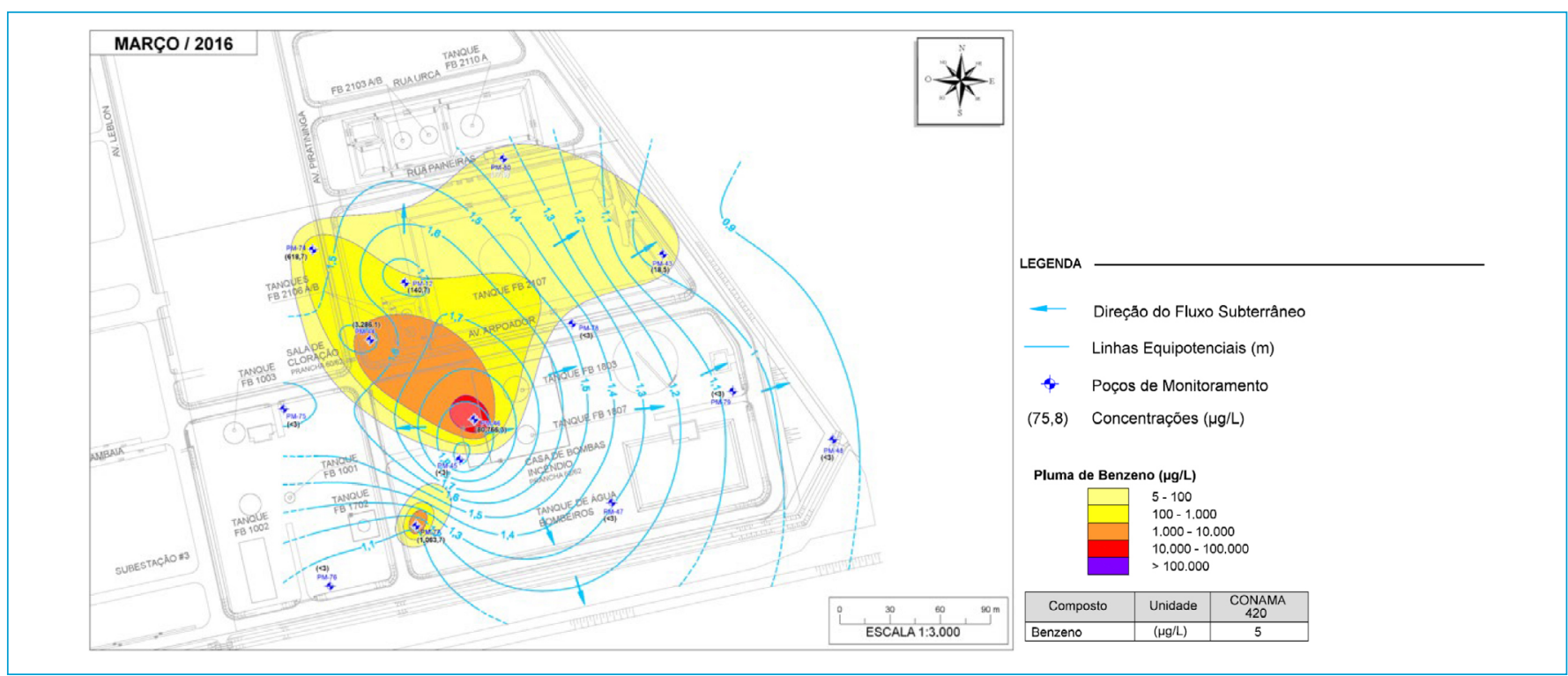

Figura 3 - Pluma de benzeno identificada na área de interesse: março de 2016.

Portanto, buscaram-se tecnologias de investigação alternativas, que diminuíssem a incerteza com relação à identificação das fontes potenciais de contaminação, e a delimitação horizontal e vertical das plumas de contaminação. Com base nos tipos de contaminantes presentes na área de interesse (i.e., principalmente BTEX) e no custo, foram aplicados os seguintes métodos de HRSC, em ordem cronológica: investigação passiva de vapores do solo com uso de amostradores PSG; e investigação com uso de MIP.

A investigação com uso do MIP é ainda uma técnica relativamente cara no Brasil, sendo o aluguel do equipamento e equipe de operação aproximadamente R 20.000 por dia, considerando a cotação do dólar na data: U\$ 1 (dólar americano) $=\mathrm{R} \$ 3,126$ (real) (BANCO CENTRAL DO BRASIL, 2017). Buscando menor tempo e custo (tendo em mente o segundo vértice da abordagem tríade), a investigação de vapores do solo foi conduzida previamente ao uso do MIP para diminuir as incertezas no entendimento da contaminação da área de interesse do estudo de caso e otimizar o uso do MIP. A investigação de vapores do solo tem um custo estimado de aproximadamente $\mathrm{R} \$ 1.000$, considerando a cotação do dólar na data: U\$ 1 (dólar americano) = R \$ 3,126 (real) (BANCO CENTRAL DO BRASIL, 2017), por amostrador (transportes Estados Unidos (EUA)/Brasil e análise).

\section{Investigação passiva de vapores do solo: trabalho de campo e resultados}

A investigação passiva de vapores do solo foi realizada utilizando-se o amostrador (sampler) passive soil gas (PSG) BeSure ("amostrador PSG"), da empresa Beacon Environmental Services Inc. (2017), composto por diversos materiais adsorventes granulares, encapsulados em uma membrana microporosa hidrofóbica, quimicamente inerte e com baixa afinidade para o vapor de água. Essa membrana permite a difusão de vapores presentes no meio, mas não permite a passagem de partículas de solo ou água.

Os amostradores PSG foram distribuídos em uma malha triangular com espaçamento de 15 por $15 \mathrm{~m}$ onde se esperava um maior impacto e no limite da área de interesse, e 20 por $20 \mathrm{~m}$ onde se esperava um impacto moderado.
Os amostradores PSG foram alocados em uma profundidade de aproximadamente $30 \mathrm{~cm}$ em sondagens com profundidade total de $1 \mathrm{~m}$ (zona não saturada) e diâmetro de 1 pol, e foram expostos aos vapores do solo por 14 dias, conforme instruções do laboratório. Esse período de tempo foi escolhido devido à presença de aterro compactado de argila pouco arenosa de até $2 \mathrm{~m}$ de profundidade em média. Cento e oitenta e nove amostradores PSG foram encaminhados para análise laboratorial da massa adsorvida. Os resultados foram reportados em valores de massa dos compostos adsorvidos. Os resultados de benzeno foram mapeados e são apresentados na Figura 4.

A partir dos resultados obtidos na investigação passiva de vapores do solo, obteve-se um delineamento horizontal da contaminação e com isso foi possível identificar os principais hotspots de contaminação e as prováveis fontes de contaminação que geraram os impactos no subsolo. Além disso, foi identificada uma possível migração para fora do site (off-site) da contaminação, que não havia sido identificada anteriormente a partir das investigações ambientais tradicionais, que é de extrema importância ambiental em razão da presença de um canal adjacente ao site, no limite leste, que desagua na Baía de Guanabara.

Observa-se que a contaminação detectada pode estar presente em solo e/ou água subterrânea e que as localizações dos hotspots de contaminação são aproximadas, pois o resultado obtido pelo amostrador PSG não reflete necessariamente a posição exata da pluma em fase dissolvida, visto que a dinâmica de migração dos voláteis na zona não saturada (vadosa) é diferente da dinâmica de migração de contaminantes presentes na zona saturada.

\section{Investigação com uso de membrane interface probe: trabalho de campo e resultados}

O plano de investigação preliminar com uso de MIP foi elaborado com base nos resultados da investigação passiva dos vapores do solo, com o intuito de complementar os resultados obtidos. A sonda utilizada neste estudo contempla três sistemas distintos: o MIP, o HPT e o EC. A sonda do MIP foi cravada no solo por meio da tecnologia direct push. Durante cada sondagem, foram coletadas e 


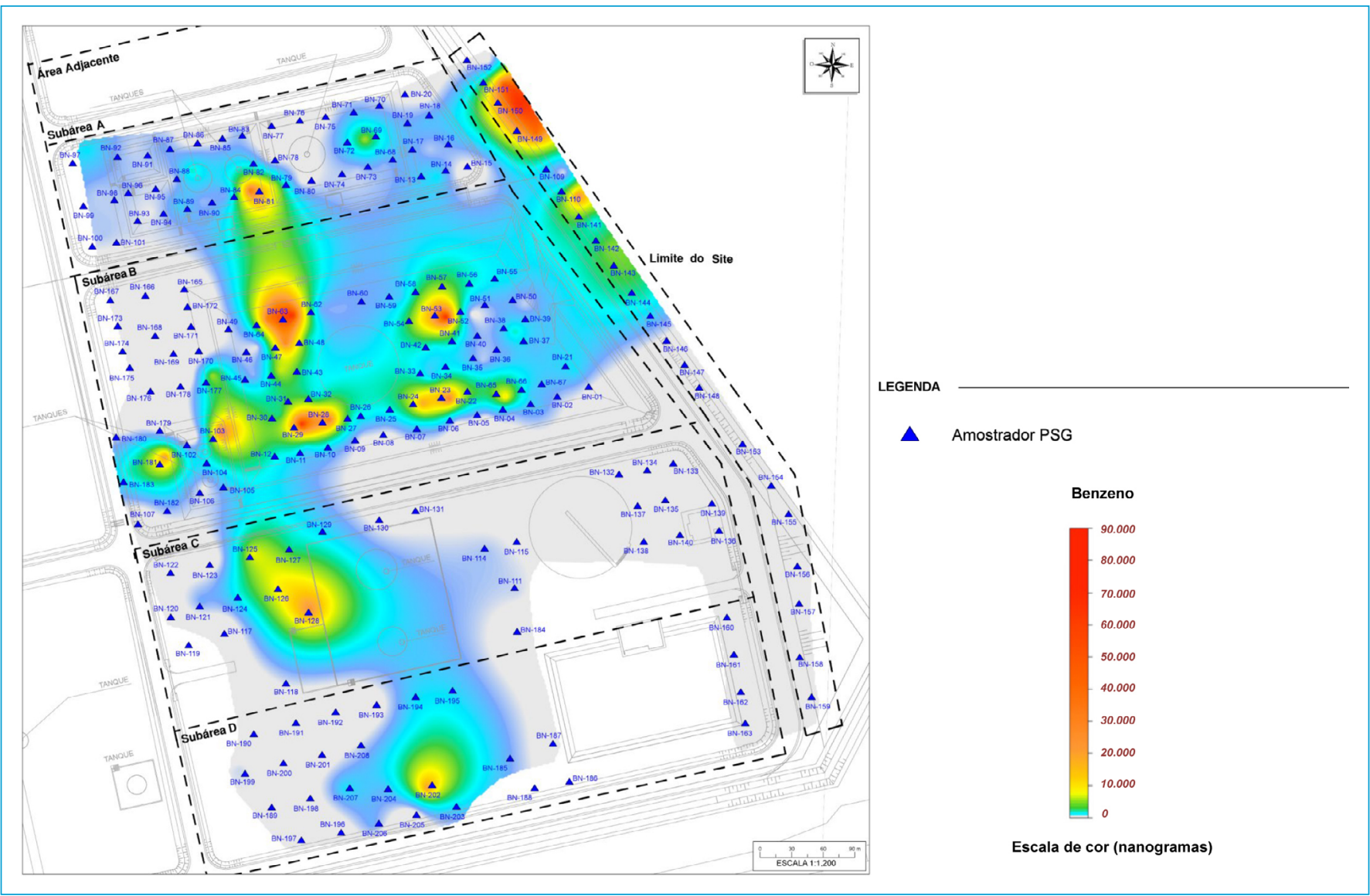

Figura 4 - Resultados da investigação passiva de vapores do solo: benzeno.

exibidas continuamente pela sonda (em tempo real) as seguintes informações: a condutividade elétrica, os parâmetros de respostas do HPT, as respostas químicas e parâmetros operacionais do MIP, a taxa de velocidade da cravação e a temperatura.

A sonda foi inserida a uma velocidade de aproximadamente $30 \mathrm{~cm}$ por minuto (equivalente a 1 pés (feet) por minuto) até alcançar a camada de argila orgânica e/ou até ser alcançada a delimitação vertical da contaminação. A profundidade variou de acordo com o ponto de sondagem e com as informações obtidas em campo. A temperatura da chapa de aço da sonda foi controlada durante as sondagens para que ela se mantivesse na faixa ótima de temperatura (i.e., de 100 a $120^{\circ} \mathrm{C}$ ).

Após a conclusão da perfilagem, as perfurações foram seladas com bentonita e cimento, da base do furo em direção ao topo, de forma a evitar possível contaminação cruzada entre diferentes profundidades no interior da sondagem, e formação de partes com espaços vazios ao longo do furo. Foram realizadas 51 sondagens.

De forma geral, a sonda foi aprofundada a uma profundidade entre 5 e $7 \mathrm{~m}$. A interpretação dos perfis de HPT e EC indicou a presença de três camadas distintas de solo (aterro, areia e argila orgânica), corroborando com as camadas identificadas durante as sondagens realizadas nas investigações tradicionais.

No momento em que a sonda atingiu a interface da camada de areia com a argila orgânica foi observado um aumento brusco nos valores de EC em todos os perfis, que foi associado às elevadas concentrações de sais encontradas na camada de argila orgânica da área, que se encontra em uma bacia sedimentar flúvio-marinha e sofre influência de uma cunha salina, e foi fundamental para determinação das profundidades das sondagens. A sondagem foi aprofundada até a próxima camada (argila marinha), dependendo das respostas obtidas na argila orgânica. Na interface da argila orgânica com argila marinha, foi observada uma diminuição nos valores de EC.

Foram realizados 32 testes de dissipação do HPT e, com base nos resultados, foi possível estimar que a profundidade inferida do nível d'água varia de 0,50 até $1,50 \mathrm{~m}$, com valor médio igual a $1,26 \mathrm{~m}$, sendo este valor consistente com valores de nível d'água medidos no site a partir dos poços de monitoramento existentes.

Durante as atividades, as detecções simultâneas no sensor PID e FID foram associadas à presença das principais substâncias químicas de interesse (SQIs) da área (i.e., BTEX) no local da sondagem, visto que elas possuem potencial de ionização inferior a 10,2 eV e diversas ligações carbono-hidrogênio (típicas de compostos orgânicos). Respostas inferiores a $0,3 \mathrm{~V}$ foram consideradas "traço" ou background.

Detecções apenas no sensor FID foram associadas à presença de metano que é um gás natural, proveniente da decomposição anaeróbica da matéria orgânica. Sua ocorrência é esperada na área em função da existência da camada de argila orgânica.

Nenhuma resposta do sensor XSD superou 0,035 V, indicando a ausência de halogenados na área, corroborando com os resultados históricos de monitoramentos de água subterrânea realizados na área. 


\section{Distribuição de compostos orgânicos voláteis na zona vadosa}

O valor médio calculado para o nível d’água $(1,26 \mathrm{~m})$ foi utilizado como o limite da zona vadosa na área de estudo. Como dito anteriormente, leituras de PID e FID na zona vadosa foram associadas à presença de BTEX. Nove sondagens apresentaram resultados significativos nos sensores PID e FID (>0,3 V), indicando um impacto na zona vadosa, e quatro apresentaram resultados considerados elevados $(>1 \mathrm{~V})$. Valores de PID maiores do que $1 \mathrm{~V}$ são um indicativo de uma possível presença de fase residual no local.

A Figura 5 apresenta as maiores leituras do sensor PID encontradas durante a investigação com uso de MIP na zona vadosa. Adicionalmente, para fins de comparação dos resultados obtidos na zona vadosa a partir de ambas as técnicas de HRSC utilizadas, os resultados obtidos de benzeno por meio da amostragem passiva de vapores do solo foram integrados com os resultados de PID na zona vadosa por meio da investigação com uso de MIP (Figura 5). Observa-se que a área a adjacente norte não foi contemplada na investigação passiva de vapores do solo, apenas na investigação com uso de MIP.

A partir da integração dos resultados da zona vadosa, observou-se que os principais hotspots identificados pela investigação passiva de vapores do solo foram confirmados com o uso de MIP.

\section{Distribuição de compostos orgânicos voláteis na zona saturada}

A análise de dados obtidos da zona saturada teve como objetivo mapear a presença de VOCs na água subterrânea, identificar potenciais caminhos preferenciais de migração de contaminantes, bem como delimitar verticalmente os impactos observados. O valor médio calculado para o nível d’água $(1,26 \mathrm{~m})$ foi utilizado para estimativa da zona saturada.

Das 51 sondagens realizadas, 25 apresentaram resultados significativos nos sensores PID e FID ( $>0,3 \mathrm{~V}$ ), e em 10 delas as leituras foram consideradas elevadas ( $>1 \mathrm{~V}$ ), indicando a presença de VOCs em fase dissolvida. Valores de PID maiores que $10 \mathrm{~V}$ foram encontrados em duas sondagens, MIP-04 (12,73 V em 1,43 $\mathrm{m}$ de profundidade) e MIP-24 (50,52 $\mathrm{V}$ em 1,62 $\mathrm{m}$ de profundidade), e são indicativos de presença de fase livre.

A Figura 6 apresenta as maiores leituras do sensor PID identificadas durante as atividades de MIP na zona saturada.

A partir da interpretação em tempo real dos resultados obtidos na investigação com uso de MIP, foi elaborado um plano de investigação complementar, com o objetivo de quantificar/confirmar os impactos observados nas investigações em alta resolução. No plano de investigação complementar, foram contempladas a instalação de nove poços (PM-84 a 90, PM-92 e PE-2) na área de interesse.

Os resultados analíticos das amostras de solo coletadas durante as atividades de instalação dos novos poços de monitoramento confirmaram o impacto identificado na zona vadosa.

Também para fins de comparação, os resultados obtidos na investigação com uso de MIP para a zona saturada (maiores leituras do sensor de PID na zona saturada) e os resultados do monitoramento de água subterrânea realizado em setembro de 2016 foram integrados e são apresentados na Figura 7.

Os resultados analíticos das amostras de água subterrânea confirmaram os resultados obtidos pela investigação com uso de MIP e indicaram presença
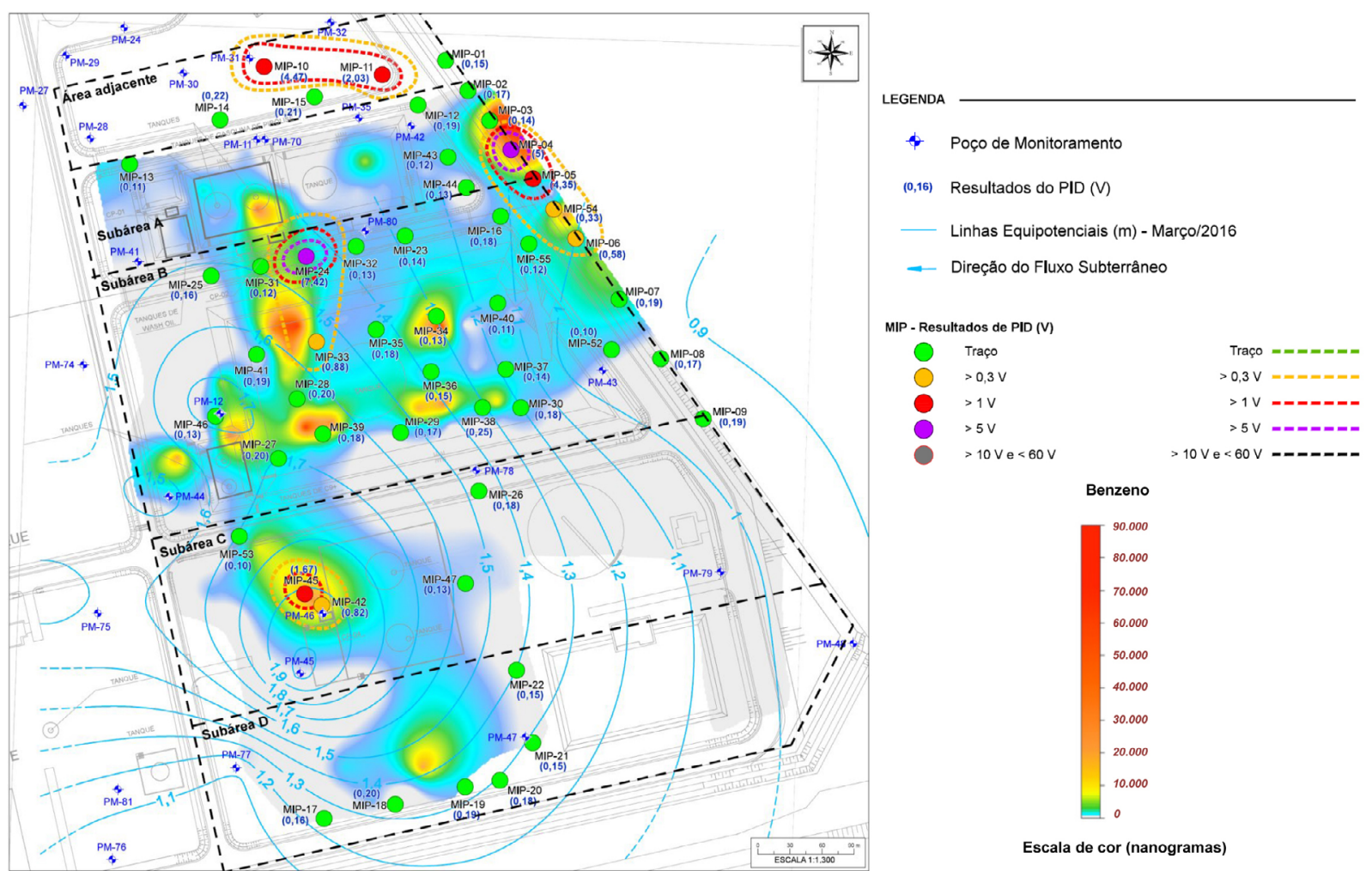

VOC: compostos orgânicos voláteis; PSG: passive soil gas; MIP: membrane interface probe.

Figura 5 - Distribuição horizontal de compostos orgânicos voláteis na zona vadosa: integração dos resultados das investigações em alta resolução (PSG e MIP). 

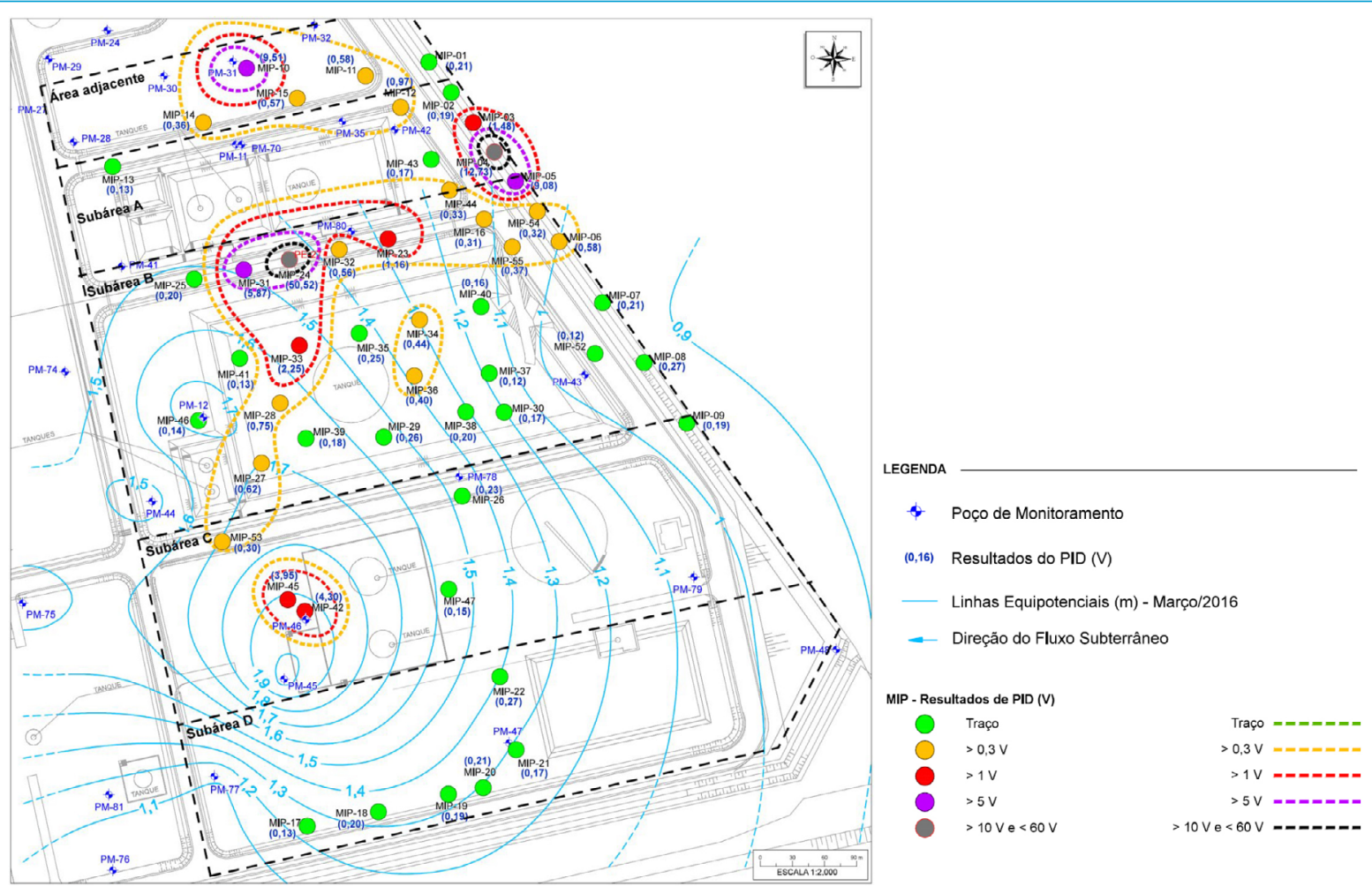

PID: detector de fotoionização

Figura 6 - Resultados de PID na zona saturada.

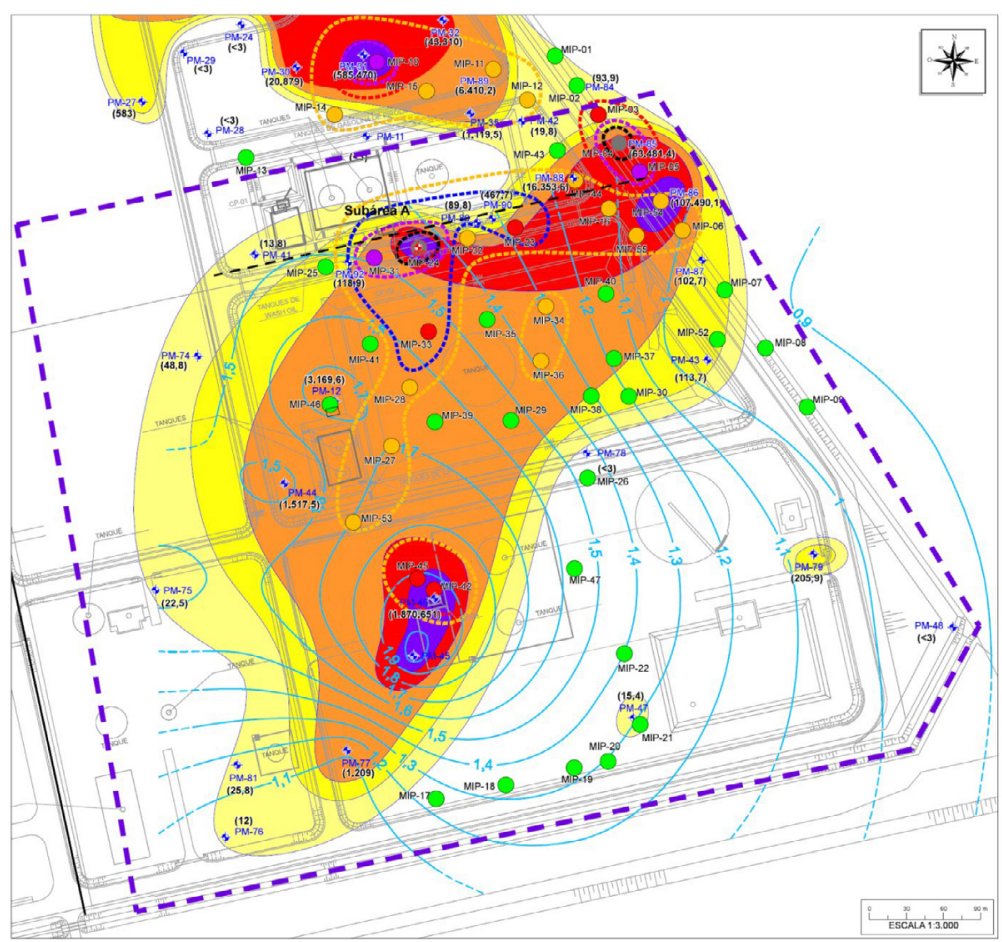

LEGENDA

Novo limite da área de interesse para fins de

gerenciamento de ACs

- Direção do Fluxo Subterrâneo

_ Linhas Equipotenciais (m)

- Poço de Extração

- Poços de Monitoramento

$(75,8)$ Concentrações $(\mu \mathrm{g} / \mathrm{L})$

Pluma de Benzeno ( $\mathrm{\mu g} / \mathrm{L})$

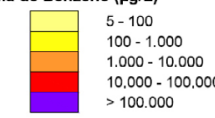

\begin{tabular}{|l|l|c|}
\hline Composto & Unidade & $\begin{array}{c}\text { CONAMA } \\
420\end{array}$ \\
\hline Benzeno & $(\mu \mathrm{g} / \mathrm{L})$ & 5 \\
\hline
\end{tabular}

MIP - Resultados de PID (V)

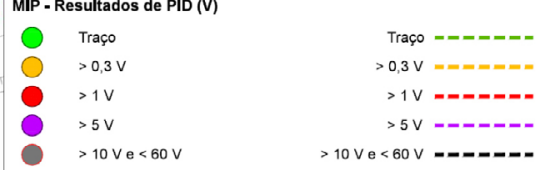

PID: detector de fotoionização; MIP: membrane interface probe.

Figura 7 - Distribuição horizontal de benzeno na zona saturada: integração dos resultados de PID na zona saturada (MIP) e do monitoramento de água subterrânea (setembro de 2016). 
de impacto na zona saturada. No poço instalado ao lado do MIP-24 (PE-27), foi identificado produto em fase livre (11 cm de espessura), compatível com os resultados de MIP, uma vez que a sondagem apresentou a detecção de PID mais elevada entre os pontos avaliados.

Os resultados integrados mostram que a distribuição de VOCs em água subterrânea ocorre de forma descontínua ao longo da área de interesse, indicando diferentes fontes de contaminação. Esse resultado foi imprescindível para priorização das próximas ações necessárias em áreas de maior preocupação ambiental, além de priorização de orçamento para os próximos passos.

Com base nos resultados integrados, foi possível agrupar centros de massas distintos de acordo com as sondagens que apresentaram leituras de PID, bem como suas correlações com os poços de monitoramento instalados, e identificar cinco hotspots e suas respectivas áreas fontes. Para ilustrar o modelo conceitual refinado, foram elaboradas seções geológicas, preparadas combinando os resultados de MIP e os resultados analíticos das amostras de água subterrâneas na amostragem de setembro de 2016.

\section{CONCLUSÕES}

O presente trabalho teve como objetivo principal utilizar diferentes metodologias de investigação, de forma complementar, para obter um modelo conceitual mais representativo de uma área de interesse.
Duas técnicas de investigação em alta resolução foram escolhidas para se alcançar um modelo conceitual que permitisse ações futuras mais eficientes, quando comparado com o que é obtido através das técnicas de investigação tradicional usualmente adotadas no estado do Rio de Janeiro.

A partir dos resultados obtidos, foi possível agrupar centros de massas distintos e identificar cinco hotspots, incluindo uma área com presença de LNAPL, que não tinha sido identificado a partir das investigações tradicionais. A detecção de sua presença e delimitação de sua extensão de forma rápida e precisa são primordiais principalmente devido ao fato de o LNAPL ser uma fonte de contaminação secundária, que, quando não identificada, permanece contaminando grandes volumes de solo e água subterrânea por longos períodos de tempo. Adicionalmente, foi identificada uma migração off-site da contaminação, de grande preocupação ambiental em razão da presença de um canal que, além de ser um bem a se proteger, desagua na Baía de Guanabara.

Com base nos dados gerados, é possível afirmar que os resultados obtidos por meio das técnicas de HRSC aplicadas foram determinantes para se obter um modelo conceitual mais representativo da área de interesse. Além disso, seria improvável obter os mesmos resultados a partir de uma investigação tradicional, nos moldes usualmente aplicados (instalando-se um número mínimo necessário de poços de monitoramento), e, para se alcançar um resultado semelhante, seria necessária a instalação de um número grande de poços de monitoramento, o que provavelmente seria realizado durante anos de trabalhos de investigação.

\section{REFERÊNCIAS}

BANCO CENTRAL DO BRASIL. Conversão de moedas. Brasil: Banco Central do Brasil, 2017. Disponível em: http://www4.bcb.gov.br/pec/conversao/ conversao.asp. Acesso em: 13 abr. 2017.

BEACON ENVIRONMENTAL SERVICES, INC. Passive Soil Gas Surveys. Beacon environmental services, 2017. Disponível em: http://www.beaconusa.com/services/passive-soil-gas-surveys/. Acesso em: 28 abr. 2017.

BRASIL. Conselho Nacional do Meio Ambiente (CONAMA). (2009). Resolução no 420. Brasil: Conselho Nacional do Meio Ambiente, 2009.

COMPANHIA AMBIENTAL DO ESTADO DE SÃO PAULO (CETESB). Diretoria de Controle de Licenciamento Ambiental. Relação de Áreas Contaminadas e Reabilitadas no Estado de São Paulo. São Paulo: CETESB, 2013.

COMPANHIA AMBIENTAL DO ESTADO DE SÃO PAULO (CETESB). Diretoria Plena. Decisão de Diretoria no 038/2017/C. São Paulo: CETESB, 2017.

CONSELHO ESTADUAL DO MEIO AMBIENTE DO RIO DE JANEIRO (CONEMA). Resolução no 44. Rio de Janeiro: CONEMA, 2012.

COUTINHO, B.R. Caracterização de áreas contaminadas através de ensaios in situ. Dissertação (Mestrado) - Departamento de Engenharia Civil, Pontifícia Universidade Católica, Rio de Janeiro, 2002.

GEOPROBE. MIP: Membrane Interface Probe. 2017. Disponível em: http://geoprobe.com/mip-membrane-interface-probe. Acesso em: 28 abr. 2017.
INSTITUTO ESTADUAL DO AMBIENTE (INEA). Instrumentos de Licenciamento. INEA, 2017. Disponível em: http://www.inea.rj.gov.br/ Portal/Agendas/LicenciamentoAmbiental/licenciamento-ambiental/ SistemadeLicenciamento/LicenaAmbiental/index.htm\&lang. Acesso em: 15 abr. 2017.

INTERNACIONAL AGENCY FOR RESEARCH ON CANCER (IARC). List of classifications. IARC, 2017. v. 1 .

KIM, J.; CORAPCIOGLU, M.Y. Modeling dissolution and volatilization of LNAPL sources migrating on the groundwater table. Journal of Contaminant Hydrology, v. 65, n. 1-2, p. 137-158, 2003. https://doi.org/10.1016/ s0169-7722(O2)00105-5

MOREIRA, C.A.; DOURADO, J.C. Análise de Contaminantes de Fase Líquida Não Aquosa (NAPLs) por aplicação de Método Eletromagnético Indutivo (EM). Revista Brasileira de Geofísica, v. 23, n. 3, p. 213-229, 2005. https://doi. org/10.1590/S0102-261X2005000300001

RIYIS, M.T.; GIACHETI,H.L.; DERRITE, R.M.; RIYIS, M.T. Investigação geoambiental de áreas contaminadas com elaboração do modelo conceitual em campo utilizando ferramentas de alta resolução (HRSC). Revista Brasileira de Geologia de Engenharia e Ambiental, v. 3, n. 1, p. 125-137, 2013.

RODRIGUES JR., J.J. Proposta Metodológica para Gerenciamento de Areas Contaminadas: uma Aplicação no Estado do Rio de Janeiro. 100f. Dissertação (Mestrado em Planejamento Energético) - Universidade Federal do Rio de Janeiro, Rio de Janeiro, 2003. 
SÁNCHEZ, L.E. A desativação de empreendimentos industriais: um estudo sobre o passivo ambiental. 178f. Tese (Doutorado Livre-Docência) - Escola Politécnica, Universidade de São Paulo, São Paulo, 1998.

SANTOS, A.D; FERREIRA, A.N.; GOBATTO, G; RIYIS, M.T. Aprimoramento do modelo conceitual da área através do uso de ferramentas de investigação emalta resolução. InterfacEHS - Saúde, Meio Ambiente e Sustentabilidade, v. 10, n. 2, 2015.

SUTHERSAN, S.; KOONS, B.; SCHNOBRICH, M. Contemporary Management of sites with Petroleum LNAPL Presence. Groundwater Monitoring \& Remediation, v. 35, n. 1, p. 23-29, 2015. https://doi.org/10.1111/gwmr.12099
UNITED STATES ENVIRONMENTAL PROTECTION AGENCY (USEPA). Best Management Practices: Use of Systematic Project Planning Under a Triad Approach for Site Assessment and Cleanup (EPA-542-F-10-010). Estados Unidos: USEPA, 2010. $18 \mathrm{p}$

UNITED STATES ENVIRONMENTAL PROTECTION AGENCY (USEPA). Summary of the Triad approach. Estados Unidos: USEPA, 2004

UNITED STATES ENVIRONMENTAL PROTECTION AGENCY (USEPA) Superfund. Estados Unidos: USEPA, 2019. Disponível em: https://www.epa. gov/superfund/what-superfund. Acesso em: 23 dez. 2019. 